PAPER

\title{
A study of stereotypic behaviours in Alzheimer's disease and frontal and temporal variant frontotemporal dementia
}

\author{
S Nyatsanza, T Shetty, C Gregory, S Lough, K Dawson, J R Hodges
}

J Neurol Neurosurg Psychiatry 2003;74:1398-1402

See end of article for authors' affiliations

....................

Correspondence to: Professor J Hodges, MRC Cognition and Brain Sciences Unit, 15 Chaucer Road, Cambridge CB2 2EF

UK; john.hodges@ mrc-cbu.cam.ac.uk

Received 15 August 2002 Accepted in final revised form 2 April 2003
Objective: To document the prevalence and pattern of stereotypic behaviour in patients with Alzheimer's dementia and frontal and temporal variants of frontotemporal dementia. Secondly, to examine the relationship between stereotypic and other neuropsychiatric behaviours.

Methods: Patients with the following were studied; Alzheimer's disease $(n=28)$, frontal variant frontotemporal dementia (fvFTD, $n=18$ ), and semantic dementia-the temporal lobe variant of FTD $(n=13)$. All patients were assessed using the Neuropsychiatric Inventory (NPI), the Mini-Mental State Examination, Addenbrooke's Cognitive Examination, and the Clinical Dementia Rating scale. Patients were also rated on the newly devised Stereotypic and Ritualistic Behaviour (SRB) subscale, which was designed as an addendum to the NPI.

Results: There was no significant difference across diagnostic groups in terms of age, sex, or severity of cognitive deficits. The overall NPI was significantly higher in patients with fvFTD compared with the other two groups, but fvFTD and semantic dementia showed a similar, and significantly increased, prevalence of stereotypic behaviours on the SRB subscale. Within the FTD group as a whole these behaviours were more likely to be complex, whereas in Alzheimer's disease, when present, such behaviours tended to be more simple stereotypies or stimulus bound repetitive behaviours. Stereotypic behaviours were not correlated with either disease severity or the extent of cognitive impairment in the fvFTD group, but were in the other two diagnostic groups.

Conclusion: Complex stereotypic behaviours are a core feature of the dementing syndrome in FTD and may reflect early and specific deficits in orbitofrontal circuitry and basal ganglia involvement.
$\mathrm{F}$ rontotemporal dementia (FTD) is the term currently used to describe a spectrum of non-Alzheimer's dementias involving the frontal and/or temporal lobes. ${ }^{12}$ FTD is a relatively common cause of dementia, accounting for about $20 \%$ of cases of dementia with pre-senile onset. ${ }^{3}{ }^{4}$ There are two principal forms, frontal variant FTD (fvFTD) and temporal variant FTD, more commonly referred to as semantic dementia. ${ }^{5}$ fvFTD presents with marked changes in personality and behaviour, including loss of insight, reduced empathy, poor self care, mood changes, change in eating patterns, disinhibition, apathy, and mental rigidity, ${ }^{2}$ while semantic dementia presents with a progressive fluent aphasia secondary to a breakdown in semantic knowledge, with preservation of speech output skills, perceptual and visuo-spatial functions, and episodic memory. ${ }^{7-10}$ Most studies have concentrated on the linguistic and cognitive deficits of semantic dementia, but more recently changes in personality and behaviour have been recognised..$^{112}$ In contrast, the early stages of Alzheimer's disease are characterised by deficits in anterograde episodic memory followed by attentional, executive, and semantic deficits, ${ }^{13}$ with personality and behaviour being relatively well preserved, at least in the early stages. ${ }^{12}$

Among the many clinical features of fvFTD described in the literature are those of ritualistic and stereotypic behaviours. These may include simple actions (grunting, hand rubbing, foot tapping); rituals relating to toileting or dressing; wandering and pacing in a fixed route; perseverative responses and stereotyped use of words and phrases ${ }^{3}$; superstitious rituals (avoiding walking on cracks in the pavement $)^{8}$; compulsive cleaning; and drinking of beverages in a specific sequence. ${ }^{14}$

A number of recent studies have begun to look in more detail at these behaviours and have confirmed that the overall prevalence of stereotypic behaviours is higher in FTD than in Alzheimer's disease, and have also suggested that the presence of such features may have discriminatory power in the diagnosis of FTD. ${ }^{12}{ }^{15}$ For instance, Bozeat et al ${ }^{12}$ examined the prevalence of a wide range of abnormal behaviours in three groups of patients (Alzheimer's disease, fvFTD, and semantic dementia), using a carer questionnaire, and found that stereotypic behaviour and changes in appetite best differentiated Alzheimer's disease and FTD. Snowden et $a l^{15}$ also found that repetitive behaviours were common in both fvFTD and semantic dementia, and suggested that these had a more compulsive quality in the latter group. Although these studies have established that patients with FTD have a high rate of stereotypic behaviours, a number of questions remain unresolved. Do patients with fvFTD and semantic dementia differ simply in terms of quantity, rather than quality, of stereotypic behaviours? Do patients with Alzheimer's disease show such behaviours at a later stage? What, if any, is the relationship between cognitive impairment and these behavioural symptoms?

The Neuropsychiatric Inventory (NPI) is now widely used in the assessment of patients with dementia syndromes and has been found to be both valid and reliable. ${ }^{17} \mathrm{~A}$ study by Levy et $a l^{18}$ found significantly higher scores for disinhibition, apathy, aberrant motor behaviour, and euphoria in patients with FTD compared with Alzheimer's disease, although it was not specified whether the FTD patients had frontal or

Abbreviations: FTD, frontotemporal dementia; fvFTD, frontal variant FTD; NIP, Neuropsychiatric Inventory; MMSE, Mini-Mental State Examination; ACE, Addenbrooke's Cognitive Examination; CDR, Clinical Dementia Rating scale; SRB, stereotypic and ritualistic behaviour 
temporal variant FTD. In addition, the NPI does not specifically contain a subscale designed to assess stereotypic behaviours, although some of these behaviours are included within the eating and aberrant motor behaviour subscales.

The aims of this study were threefold. Firstly, to develop a simple assessment instrument as an adjunct to the NPI to assess stereotypical and repetitive behaviours. Secondly, to document the prevalence and profile of stereotypic behaviours in patients with Alzheimer's disease, fvFTD, and semantic dementia. Thirdly, to examine the relationship between stereotypic and other neuropsychiatric behaviours and markers of cognitive decline.

\section{METHODS}

\section{Participants}

A total of 59 patients participated in the study: 28 with a diagnosis of Alzheimer's disease, (14 male and 14 female, with a mean age of 61.6, SD 5.8), 18 patients with fvFTD ( 15 male and 3 female, mean age 61.1, SD 5.8), and 13 with semantic dementia (7 male and 6 female, mean age 66.5, SD 4.4). All patients had attended the Early Onset Dementia Clinic at Addenbrooke's Hospital, Cambridge, UK, between December 1998 and December 2000. The study was approved by the Cambridge local ethics committee and all subjects gave written informed consent to participate.

All patients in the FTD group met the consensus criteria for FTD $^{1}$ and were subcategorised into those with semantic dementia or fvFTD based on previous criteria. ${ }^{9} 1920$ In brief, those with semantic dementia presented with progressive fluent aphasia with evidence of impaired comprehension in the context of preserved non-linguistic abilities and anterior temporal lobe atrophy. Patients with fvFTD had predominant changes in personality and social behaviour with evidence of frontal executive dysfunction and/or frontal atrophy on MRI. The diagnosis of Alzheimer's disease was made according NINCDS-ADRDA criteria. ${ }^{21}$

All patients were assessed by a senior neurologist, psychiatrist, and a clinical neuropsychologist. Major psychiatric illnesses (such as depression and schizophrenia) were excluded after clinical assessment and following the administration of standard psychiatric rating scales. ${ }^{22}$ Participants with a history of head trauma, alcoholism, movement disorder, or any other condition known to impair frontal lobe function were also excluded. All underwent a battery of routine screening blood tests and structural imaging (CT or MRI brain scan). Approximately half of the subjects participated in an earlier study assessing a broad range of neurobehavioural symptoms in FTD and Alzheimer's disease. ${ }^{12}$

\section{Procedure}

Cognitive function was assessed using the Mini-Mental State Examination (MMSE) ${ }^{23}$ and the Addenbrooke's Cognitive Examination (ACE). ${ }^{24}$ The ACE is a bedside schedule that incorporates the MMSE, but expands tests of memory, language, visuo-spatial function, and adds tests of verbal fluency. Neuropsychological assessment was performed by an experienced neuropsychologist. A general assessment of the severity of the dementia was made using the Clinical Dementia Rating scale (CDR). ${ }^{25}$ The CDR rates six behavioural and cognitive categories consisting of memory, orientation, judgement and problem solving, community affairs, home and hobbies, and personal care on a scale of 0-3 from information provided by carers.

Based upon a prior survey of abnormal behaviours in FTD ${ }^{12}$ and examination of the literature, we devised the stereotypic and ritualistic behaviours (SRB) subscale as an addendum to the NPI. The subscale assesses a number of areas: (a) the use of a stock phrase (verbal stereotypies)

(b) echolalia and/or verbal perseveration

(c) rigid adherence to routines/rituals

(d) preoccupation with counting

(e) clock watching

(f) repetitively pursuing the same leisure activities

(g) hoarding or collecting obsessively

(h) repetitively eating the same food (see appendix)

The SRB subscale was given simultaneously with the NPI and scored in the same way, that is, the product of the frequency of the behaviour (rated on a scale of 1-4), and the severity of the behaviour (rated on a scale of 1-3). The sum of the 13 subscale scores yielded the total NPI score.

To establish the reliability of the instrument we administered the NPI including the SRB subscale to a subgroup of 20 patients who attended the clinic on two occasions separated by no more than a month. Scores across the two occasions were highly consistent.

\section{Statistical analysis}

In view of the skewed distribution of data we employed nonparametric statistics (Kruskal-Wallis) to compare differences across three groups and Mann-Whitney rank sum test to compare differences between two groups. A principal component analysis was used to explore the structure of the NPI. The prevalence of the individual items of the SRB subscale across the three groups was assessed using Fisher's exact test, with post hoc pair wise comparisons. Correlations between the SRB subscale score and the NPI, MMSE, ACE, and CDR scores were examined using the Spearman's rank correlation coefficient.

\section{RESULTS}

The demographic details of the patient groups are shown in table 1. There was no significant difference between the groups in terms of age or sex. Although there were more males in the fvFTD group, this was not statistically significant. Similarly, there was no significant difference between the groups in their scores on the MMSE, ACE, and CDR.

Comparison of the total NPI scores revealed a highly significant group difference $(p=0.001)$. Post hoc analysis confirmed significantly higher scores in the fvFTD group, in comparison with both the Alzheimer's disease and the semantic dementia groups, with no difference between the latter two groups (fvFTD $>$ semantic dementia $=$ Alzheimer's disease). Analysis of the stereotypic behaviours subscale score also revealed a significant intergroup difference $(\mathrm{p}<0.001)$, but with a different pattern of performance across the three groups: fvFTD $=$ semantic dementia $>$ Alzheimer's disease. The distribution of the NPI subscale scores, in each of the three groups, is as shown in table 2.

A principal component analysis revealed a four factor solution accounting for $61 \%$ of the overall variance (see table 3). A single large factor accounted for $35.7 \%$ of the total variance, and comprised nine of the NPI subscales (SRB, agitation, aberrant motor behaviour, eating/appetite change, disinhibition, anxiety, apathy, euphoria, and irritability/ lability), with the maximum loading from the SRB subscale. The depression subscale alone accounted for the second factor. The subscales of delusions and hallucinations contributed together towards the third factor. Abnormal night time behaviour was the fourth and smallest factor.

The stereotypic behaviours subscale score did not correlate with age $(r=-0.03, \mathrm{p}=0.8)$ or sex $(z=-1.05, \mathrm{p}=0.3)$. Analysis of individual items in the subscale revealed two distinct patterns in the distribution of the scores for 
Table 1 Demographic and clinical variables of patients groups

\begin{tabular}{|c|c|c|c|c|}
\hline & \multicolumn{4}{|c|}{ Diagnostic group } \\
\hline & $A D(n=28)$ & fvFTD $(n=18)$ & SD $(n=13)$ & p Value \\
\hline $\begin{array}{l}\text { Age (median) in years } \\
\text { (IQR) }\end{array}$ & $61.0(9.0)$ & $60.0(8.0)$ & $64.5(7.8)$ & $p=0.14$ \\
\hline $\operatorname{Sex}(m / f)$ & $14 / 14$ & $15 / 3$ & $7 / 6$ & $p=0.29$ \\
\hline MMSE (IQR) & $21.0(12.0)$ & $22.0(10.0)$ & $20.0(18.8)$ & $p=0.8$ \\
\hline ACE (IQR) & $62.0(39.0)$ & $75.0(30.0)$ & $52.5(53.0)$ & $p=0.10$ \\
\hline CDR (IQR) & $1.0(1.5)$ & $2.0(2.5)$ & $1.0(1.6)$ & $p=0.23$ \\
\hline Total NPI score (IQR) & $12.0(15.0)$ & $59.0(30.0)$ & $15.0(29.0)$ & $p=0.001 \mathrm{fvFTD}>S D=A D$ \\
\hline SRB subscale score (IQR) & $0(3)$ & $6(8)$ & $4(10)$ & $\mathrm{p}<0.001 \mathrm{fvFTD}=\mathrm{SD}>\mathrm{AD}$ \\
\hline \multicolumn{5}{|c|}{$\begin{array}{l}\text { Median values. } \\
\text { IQR, inter quartile range; AD, Alzheimer's disease; fvFTD, frontal variant frontotemporal dementia; SD, semantic } \\
\text { dementia; MMSE, Mini-Mental State Examination; ACE, Addenbrooke's Cognitive Examination; CDR, Clinical } \\
\text { Dementia Rating scale; NPI, Neuropsychiatric Inventory; SRB, stereotypic and ritualistic behaviours. }\end{array}$} \\
\hline
\end{tabular}

individual items across the three groups (see table 4). The first cluster of symptoms: preoccupation with counting and/ or clock watching; consistently choosing the same leisure activity or hobby; repetitively eating the same food; and rigid adherence to routine, were all more common in the two FTD subgroups than in the Alzheimer's disease group, and many were virtually absent in the latter group. In contrast, the use of stock phrases (verbal stereotypies), echolalia, and/or verbal perseveration was equally common in all three groups. For collecting/hoarding, the rate was equivalent in fvFTD and Alzheimer's disease, but the difference across the groups failed to reach significance $(p=0.09)$.

Table 5 shows the correlation between the SRB subscale score, the total NPI score, and MMSE, ACE, and CDR, scores for the total study group and for each group of patients. The SRB subscale score was significantly correlated with the total NPI score, in the group as a whole and also in each of the three groups. Correlation with the degree of cognitive impairment, as measured by the MMSE or the ACE scores, reached significance only in the semantic dementia group, being absent or weak in the other two groups. In contrast, there was a stronger association between the SRB subscale score and the level of dementia, as measured by the CDR score, which reached significance in both semantic dementia and Alzheimer's disease groups, but not in the fvFTD group.

Table 3 Principal component analysis showing loading of the Neuropsychiatric Inventory (NPI) subscales, eigen values, and variance accounted for by each factor

\begin{tabular}{|c|c|c|c|c|}
\hline & Factor 1 & Factor 2 & Factor 3 & Factor 4 \\
\hline Eigen value & 4.64 & 1.70 & 1.66 & 1.2 \\
\hline$\%$ Variance & 35.7 & 13.1 & 12.8 & 9.1 \\
\hline $\begin{array}{l}\text { Stereotypic and } \\
\text { ritualistic behaviour }\end{array}$ & 0.77 & - & - & - \\
\hline Agitation & 0.72 & - & - & - \\
\hline $\begin{array}{l}\text { Aberrant motor } \\
\text { behaviour }\end{array}$ & 0.73 & - & - & - \\
\hline $\begin{array}{l}\text { Appetite/eating } \\
\text { change }\end{array}$ & 0.70 & - & - & - \\
\hline Disinhibition & 0.67 & - & - & - \\
\hline Anxiety & 0.65 & 0.48 & - & - \\
\hline Apathy & 0.64 & - & - & - \\
\hline Euphoria & 0.63 & - & - & - \\
\hline Irritability & 0.62 & 0.37 & - & - \\
\hline Depression & - & 0.76 & - & - \\
\hline Delusions & 0.44 & - & 0.78 & - \\
\hline Hallucinations & 0.45 & - & 0.81 & - \\
\hline Night time behaviour & - & 0.30 & - & 0.81 \\
\hline
\end{tabular}

Values below 0.3 removed.
Table 2 Neuropsychiatric Inventory (NPI) subscale scores in patients with Alzheimer's disease (AD), frontal variant frontotemporal dementia (fvFTD), and semantic dementia (SD)

\begin{tabular}{llll}
\hline \multicolumn{4}{c}{ Diagnostic group } \\
\cline { 2 - 4 } & $\begin{array}{l}\text { ADn= 28 } \\
\text { Median (IQR) }\end{array}$ & $\begin{array}{l}\text { fvFTDn }=18 \\
\text { Median (IQR) }\end{array}$ & $\begin{array}{l}\text { SDn = 13 } \\
\text { Median (IQR) }\end{array}$ \\
\hline Delusions & $0.6(2.3)$ & $2.4(4.2)$ & $0.0(0.0)$ \\
Hallucinations & $0.7(1.9)$ & $1.7(3.2)$ & $0.0(0.0)$ \\
Agitation & $1.2(2.6)$ & $4.6(4.3)$ & $2.4(3.6)$ \\
Depression & $1.6(2.8)$ & $2.0(3.7)$ & $2.0(3.3)$ \\
Anxiety & $1.6(2.9)$ & $2.7(4.2)$ & $1.2(2.4)$ \\
Euphoria & $0.9(1.6)$ & $2.4(4.1)$ & $1.0(1.9)$ \\
Apathy & $3.8(4.1)$ & $7.9(4.3)$ & $3.9(4.0)$ \\
Disinhibition & $0.9(2.0)$ & $6.0(4.2)$ & $2.7(3.3)$ \\
Irritability & $1.0(2.2)$ & $4.8(4.6)$ & $1.5(3.6)$ \\
Aberrant motor behaviour & $2.2(3.9)$ & $4.0(4.7)$ & $3.0(4.7)$ \\
Night time behaviour & $1.2(3.2)$ & $1.3(2.4)$ & $0.9(1.6)$ \\
Appetite/eating & $1.8(2.7)$ & $6.8(4.8)$ & $2.9(4.4)$ \\
Stereotypic behaviour & $1.6(2.6)$ & $6.6(4.3)$ & $5.7(4.9)$ \\
\hline IQR, inter quartile range. & & & \\
\hline
\end{tabular}

\section{DISCUSSION}

This study confirms previous reports that stereotypic and ritualistic behaviours occur significantly more frequently in patients with fvFTD and semantic dementia compared with Alzheimer's disease. ${ }^{14-16}$ Two distinct patterns were seen. Complex ritualised behaviours (preoccupation with counting

Table 4 Stereotypic and ritualistic behaviours subscale scorest in patients with Alzheimer's disease (AD), frontal variant frontotemporal dementia (fvFTD), and semantic dementia (SD)

\begin{tabular}{|c|c|c|c|c|}
\hline & $A D$ & fvFTD & SD & $\chi^{2}$ Value \\
\hline $\begin{array}{l}\text { Choosing the same leisure } \\
\text { activity/hobby }\end{array}$ & $3.6 \%$ & $27.8 \%$ & $46.2 \%$ & $11.07^{* *} \dagger$ \\
\hline $\begin{array}{l}\text { Clock watching/preoccupation } \\
\text { with time }\end{array}$ & $3.6 \%$ & $33.3 \%$ & $38.5 \%$ & $10.10^{* *} \dagger$ \\
\hline Preoccupation with counting & $0 \%$ & $22.2 \%$ & $30.8 \%$ & $9.77^{* *} \dagger$ \\
\hline Rigid adherence to routines & $14.3 \%$ & $50.0 \%$ & $30.8 \%$ & $6.71 *+$ \\
\hline $\begin{array}{l}\text { Repetitively eating the same } \\
\text { food }\end{array}$ & $0 \%$ & $5.6 \%$ & $15.4 \%$ & $6.88^{*} \ddagger$ \\
\hline $\begin{array}{l}\text { Hoarding or collecting } \\
\text { obsessively }\end{array}$ & $14.3 \%$ & $27.8 \%$ & $0 \%$ & 4.25 (NS) \\
\hline Verbal perseveration & $10.7 \%$ & $33.3 \%$ & $15.4 \%$ & 3.54 (NS) \\
\hline Use of a stock phrase & $21.4 \%$ & $38.9 \%$ & $46.2 \%$ & 3.09 (NS) \\
\hline
\end{tabular}

†Percentage of patients in each of the three groups scoring $>0$ on each item. $¥$ Post hoc analysis $S D=f v F T D>A D$. $¥ S D>f v F T D=A D$. ${ }^{*} \mathrm{p}<0.05 ;{ }^{* *} \mathrm{p}<0.005$. 
Table 5 Association between the SRB subscale score and the total NPI, MMSE, ACE, and CDR score using Spearman's rank

\begin{tabular}{lccrc}
\hline & Total Group & \multicolumn{1}{c}{ AD } & \multicolumn{1}{c}{ fvFTD } & \multicolumn{1}{c}{ SD } \\
\hline Total NPI score & $0.73^{* *}$ & $0.48^{*}$ & & $0.94^{* *}$ \\
MMSE score & -0.12 & -0.27 & -0.02 & $-0.75^{*}$ \\
ACE score & -0.18 & -0.28 & -0.08 & $-0.75^{*}$ \\
CDR score & $0.48^{* *}$ & $0.53^{*}$ & 0.35 & $0.72^{*}$ \\
\hline
\end{tabular}

$\mathrm{p}<0.05 ;{ }^{* *} \mathrm{p}<0.01 ;{ }^{* * *} \mathrm{p}<0.001$

SRB, stereotypic and ritualistic behaviours; $A D$, Alzheimer's disease; fvFTD, frontal variant frontotemporal dementia; SD, semantic dementia; MMSE, Mini Mental State Examination; ACE, Addenbrooke's Cognitive Examination; CDR, Clinical Dementia Rating Scale; NPI,

Neuropsychiatric Inventory.

and/or clock watching, consistently choosing the same leisure activity or hobby, repetitively eating the same food, and rigid adherence to routine) occurred significantly more frequently in patients with fvFTD and semantic dementia than in Alzheimer's disease. In contrast, simpler verbal stereotypies/ perseveration or stimulus bound behaviour, such as echolalia, were equally common across the three groups.

The results of the principal component analysis indicate that there are four distinct clusters of neurobehavioural symptoms in these patient groups. One single factor accounted for $36 \%$ of the total variance. This included stereotypic and ritualistic behaviours as well as agitation, aberrant motor behaviour, eating/appetite change, disinhibition, anxiety, apathy, euphoria, and irritability/lability. Interestingly, depression, delusions/hallucinations, and disturbed night time behaviour were all separate factors, suggesting a distinct neural basis for these features. These findings mirror those reported by Bozeat et al ${ }^{12}$ who, using a carer based questionnaire, found a similar pattern.

Within the fvFTD subgroup, the severity of stereotypic behaviours did not correlate with the level of dementia as assessed by either cognitive measures (MMSE and ACE), or more global functional assessment, the CDR. This suggests that stereotypic and ritualistic behaviours are a core feature of fvFTD, occurring early in the course of the illness and reflecting specific disruption of neural circuitry. In semantic dementia and Alzheimer's disease there was a clearer association between the total NPI score and disease severity, suggesting that changes in behaviour reflect advancing disease. This finding is supported by Ames et $\mathrm{al}^{26}$ who reported that complex ritualistic behaviours are typically observed early in the course of frontal lobe degeneration, whereas elementary stereotyped and repetitive behaviours are seen in late stage patients.

The finding of an essentially similar profile of SRBs in fVFTD and semantic dementia offers further evidence that these two syndromes represent manifestations of the same underlying frontotemporal disorder. This is somewhat at odds with the recent study by Snowden et $\mathrm{al}^{15}$ in which patients with semantic dementia had significantly more compulsive symptoms (particularly clock watching, orderliness, and adherence to routine) as compared with those with fvFTD. Snowden et al divided their fvFTD patient group into disinhibited (pseudopsychopathic) and apathetic patients; the latter showed a marked tendency towards simple repetitive behaviours, while disinhibited FTD patients and patients with semantic dementia exhibited both simple and complex repetitive behaviours.

It is known that in fvFTD the brunt of the pathology involves the orbital (ventromedial) frontal lobe, while in semantic dementia the temporal pole and amygdala are severely atrophic. ${ }^{5}$ A number of authors have suggested that the triad of disinhibition, stereotypic behaviours, and gluttony, which characterises FTD, reflects disruption of an integrated circuit involving the orbitofrontal cortex, amygdale, and the insular. ${ }^{56}$ In addition to focal cortical atrophy, there is evidence of striatal pathology in FTD, which Neary et $\mathrm{al}^{3}$ speculate might be relevant to the genesis of stereotypic and ritualistic behaviours. However, without parallel structural and functional imaging it is not possible to resolve the issue of whether the complex stereotypic and ritualistic behaviours found in FTD, but not in Alzheimer's disease, reflect pathological involvement of the orbitofrontalamygdala-insular network or, alternatively, primary striatal pathology.

One potential limitation of our study is that the patients were selected from a tertiary specialist referral centre, which may have lead to an inherent sampling bias. However, balanced against this is the comprehensive assessment performed in the clinic that allows relatively pure examples of each diagnostic category to be studied.

In conclusion, the SRB subscale appears to be a useful tool for differentiating the frontal and temporal variants of FTD and Alzheimer's disease. It is hoped that incorporation of this subscale in the NPI could extend its discriminatory power and relevance in clinical and research settings.

\section{ACKNOWLEDGEMENT}

The statistical analysis was conducted with the help of Miss Sarah L Vowler and Dr A T Prevost, from The Centre for Applied Medical Statistics, Department of Public Health and Primary Care, University of Cambridge.

The research was supported by a Medical Research Council programme grant to JRH number G9724461.

\section{Authors' affiliations}

S Nyatsanza, C Gregory, S Lough, Psychiatric services for the elderly, Fulbourn Hospital, Cambridge, UK

T Shetty, K Dawson, Department of Neurology, Addenbrooke's NHS

Trust, Cambridge CB2 2QQ, UK

J R Hodges, MRC Cognition and Brain Sciences Unit, Chaucer Road, Cambridge CB2 2EF, UK

Competing interests: none declared

\section{APPENDIX}

Addendum to NPI: stereotypic and ritualistic behaviours Has he/she become more rigid and fixed his/her opinions? Has he/she developed a repetitive phrase or tendency to repeat questions? Has he/she developed routines, which cannot easily be discouraged? Has he/she seemed preoccupied with counting numbers or clock watching?

NO (if no, proceed to next screening question). YES (If yes, proceed to subquestions)

- Has he/she developed a repetitive phrase that he/she uses often?

- Does he/she repeat a question before replying to it, or repeat an answer numerous times?

- Has he/she developed routines that cannot be easily discouraged, such as taking exactly the same routes around the home or garden, needing to do household tasks in exactly the same order, or

- Does he/she seem preoccupied with counting things with numbers?

- Does he/she clock watch or seem pre-occupied by time?

- Does he/she consistently choose to do the same leisure activities or hobbies such as jigsaw puzzles or word searches?

- Does he/she hoard or collect things obsessively? If so, what kind of things? 
- Does he/she want to eat exactly the same food repeatedly? (NB: This question is also in the Appetite and Eating Disorders Section).

If the screening question is confirmed, determine the frequency and severity of the stereotypical ritualistic behaviour.

Frequency:

1. Often - about once a week

2. Occasionally - less than once a week

3. Frequently - several times per week but less than every day

4. Very frequently - once or more per day or continuously. Severity:

1. Mild - changes in stereotypic and ritualistic behaviours are present but are not disturbing

2. Moderate - changes in stereotypic and ritualistic behaviour are present and cause minor disturbance

3. Marked - obvious changes in stereotypic and ritualistic behaviours are present and cause disturbance and upset the patient.

Distress: How emotionally distressing do you find this behaviour?

Not at all

Minimally

Mildly

Moderately

Severely

Very severely or extremely.

\section{REFERENCES}

1 The Lund and Manchester Groups. Clinical and neuropathological criteria for frontotemporal dementia. J Neurol Neurosurg Psychiatry 1994;57:416-8.

2 Gregory CA, Hodges JR. Frontotemporal dementia: use of consensus criteria and prevalence of psychiatric features. Neuropsychiatry Neuropsychol Behav Neurol 1996:9:145-53.

3 Neary D, Snowden JS, Mann DM. Classification and description of frontotemporal dementias. Ann N Y Acad Sci 2000;920:46-51.

4 Harvey RJ. Epidemiology of presenile dementia. In: Hodges JR, ed. Earlyonset dementia: a multidisciplinary approach. Oxford: Oxford University Press, 2001:1-23.

5 Hodges JR, Miller B. The neuropsychology of frontal variant frontotemporal dementia and semantic dementia. Introduction to the special topic papers: Part II. Neurocase 2001;7:113-21.
6 Cummings JL, Benson DF. Dementia: a clinical approach. Boston: Butterworth, 1992.

7 Snowden JS, Goulding PJ, Neary D. Semantic dementia: a form of circumscribed cerebral atrophy. Behav Neurol 1989;2:167-82.

8 Snowden JS, Neary D, Mann DMA, In: Gardner L, Singleton P, eds. Frontotemporal lobar degeneration: fronto-temporal dementia, progressive aphasia, semantic dementia. New York: Churchill Livingstone, 1996.

9 Hodges JR, Patterson K, Oxbury S, et al. Semantic dementia: progressive fluent aphasia with temporal lobe atrophy. Brain 1992;115:1783-806.

10 Hodges JR, Patterson K, Tyler LK. Loss of semantic memory: implications for the modularity of mind. Cognit Neuropsychol 1994;11:505-42.

11 Edwards Lee T, Miller B, Cummings J, et al. The temporal lobe variant of frontotemporal dementia. Neurology 1996;46(Suppl. 1):A178.

12 Bozeat S, Gregory CA, Lambon Ralph MA, et al. Which neuropsychiatric and behavioural features distinguish frontal and temporal variants of frontotemporal dementia from Alzheimer's disease? J Neurol Neurosurg Psychiatry 2000;69:178-86.

13 Hodges JR, Patterson K, Ward P, et al. The differentiation of semantic dementia and frontal lobe dementia (temporal and frontal variants of frontotemporal dementia) from early Alzheimer's disease: a comparative neuropsychological study. Neuropsychology 1999;13:31-40

14 Miller BL, Darby AL, Swartz JR, et al. Dietary changes, compulsions and sexual behavior in frontotemporal degeneration. Dementia 1995:6:195-9.

15 Snowden JS, Bathgate D, Varma A, et al. Distinct behavioural profiles in frontotemporal dementia and semantic dementia. J Neurol Neurosurg Psychiatry $2001 ; 70: 323-32$

16 Shigenobu K, Ikeda M, Fukuhara R, et al. The Stereotypy Rating Inventory in Frontotemporal lobar degeneration. Psychiatry Res 2002;110:175-87.

17 Cummings JL, Mega M, Gray K, et al. The Neuropsychiatric Inventory: comprehensive assessment of psychopathology in dementia. Neurology 1994:44:2308-14.

18 Levy ML, Miller BL, Cummings JL, et al. Alzheimer disease and frontotemporal dementias: behavioral distinctions. Arch Neurol 1996;53:687-90.

19 Gregory CA, Hodges JR. Dementia of frontal type and the focal lobar atrophies. Int Rev Psychiatry 1993:5:397-406.

20 Gregory CA, Hodges JR. Clinical features of frontal lobe dementia in comparison with Alzheimer's disease. J Neural Trans 1996:47:103-23.

21 McKhann G, Drachman D, Folstein M, et al. Clinical diagnosis of Alzheimer's disease report of the NINCDS-ADRDA work group under the auspices of Department of Health and Human Services Task Force on Alzheimer's disease. Neurology 1984;34:939-44.

22 Hodges JR, Berrios GE, Breen K. Memory disorders in psychiatric pactice. In: Berrios GE, Hodges JR, eds. The multidisciplinary memory clinic approach. Cambridge: Cambridge University Press, 2000:101-21.

23 Folstein MF, Folstein SE, McHugh PR. 'Mini-Mental State': a practical method for grading the cognitive state of patients for the clinician. J Psychiatr Res 1975;12:189-98.

24 Mathuranath PS, Nestor P, Berrios GE, et al. A brief cognitive test battery to differentiate Alzheimer's disease and frontotemporal dementia. Neurology 2000;55:1613-20.

25 Hughes CP, Berg L, Danziger WL, et al. A new clinical scale for the staging of dementia. Br J Psychiatry 1982;140:566-72.

26 Ames D, Cummings JL, Wirshing WC, et al. Repetitive and compulsive behavior in frontal lobe degenerations. I Neuropsychiatry Clin Neurosci 1994;6: 100-13. 